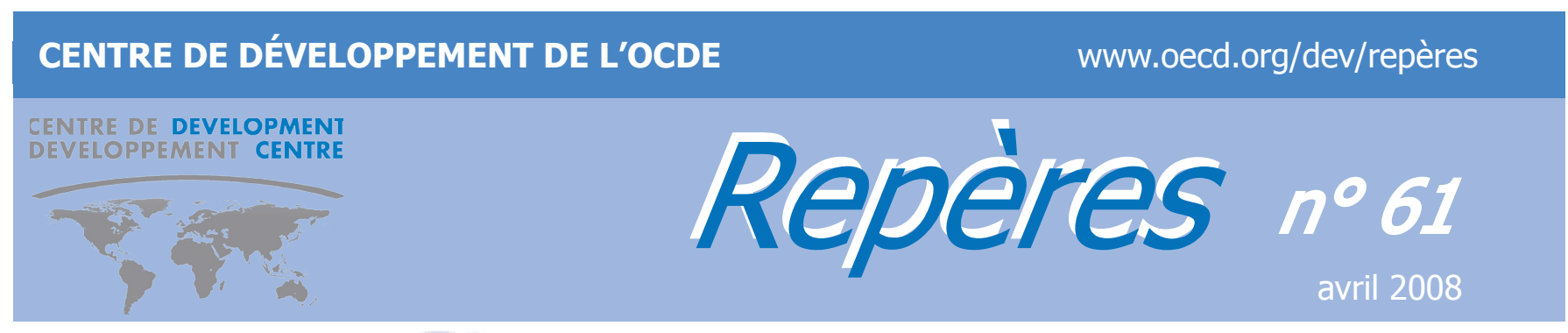

\title{
Évaluer les effets des formations professionnelles en Afrique
}

\author{
(Rédigé à partir des Perspectives économiques en Afrique 2008*) \\ par Christian Kingombe
}

- Une amélioration quantitative et qualitative des données est nécessaire pour évaluer l'impact des formations professionnelles et techniques sur la croissance économique et la réduction de la pauvreté.

- Les observatoires du marché du travail peuvent aider à aligner les systèmes de formation aux besoins du marché du travail.

- L'analyse du chômage des jeunes est essentielle avant tout investissement dans des programmes de formation onéreux.

On ne connaît pas l'impact des formations professionnelles sur la croissance économique et la réduction de la pauvreté dans les pays africains. Il est difficile pour les pays et les donneurs de formuler des politiques en l'absence de ces informations. Même les 35 pays qui ont été sondés dans le rapport Perspectives économiques en Afrique (PEA) 2008 n'ont été en mesure que de fournir des données approximatives ${ }^{1}$.

Le développement des compétences techniques et professionnelles en Afrique intervient à différents niveaux de l'éducation. Les gouvernements et les donneurs doivent promouvoir le mélange des compétences qui correspond le mieux aux exigences des pays et à leur stade de développement économique. De plus, les politiques qui visent à encourager le développement des compétences et les formations professionnelles sont plus efficaces lorsqu'elles sont en accord avec les politiques de développement globales et les besoins du marché du travail.

Par conséquent, il devient essentiel d'analyser l'apport des formations professionnelles au développement d'un pays. Ceci permet non seulement aux gouvernements et aux ONG de sélectionner les programmes qui contribuent le plus au développement individuel et national mais apporte aussi un moyen permettant aux parties prenantes, en particulier les donneurs, de percevoir la valeur de leur contribution. Une bonne analyse des programmes et des

1. Le rapport intitulé Perspectives économiques en Afrique (PEA) est une publication annuelle de la Banque africaine de développement et le Centre de développement de l'OCDE. politiques de formation professionnelle tire les leçons des expériences passées et évalue leur impact sur les vies et les carrières des bénéficiaires. L'impact de ces formations peut ainsi être évalué dans le contexte général, ce qui inclue les ressources naturelles, le milieu des affaires local, la disponibilité des équipements, la demande, et l'accès au marché. Si tous ces éléments sont pris en compte, les services, la planification et l'allocation des ressources devraient s'améliorer.

L'analyse des programmes de formation dans le secteur informel en Côte d'Ivoire entre 1994 et 2002 indique ceux qui ont produit des effets économiques positifs ; au Bénin, une étude sur la qualité des programmes entre 2000 et 2005 a mis en évidence des résultats positifs possibles, puisque 60 pour cent des participants ont affirmé que leur chiffre d'affaire et leurs bénéfices avaient augmenté en même temps que les coûts avaient baissé.

L'importance de la formation n'est donc pas en doute.

L'amélioration de l'employabilité de la force de travail, via un accroissement des compétences, dépend non seulement de la qualité des compétences en elles-mêmes, mais aussi du dynamisme du contexte économique dans lequel elles peuvent être déployées et de la disponibilité des formations. Les gouvernements peuvent mieux contrôler la qualité de l'offre de formation privée en optimisant I'utilisation des institutions privées en complémentarité aux programmes financés par l'État. Cela s'est notamment avéré efficace en Zambie. 
Les mécanismes de contrôle et d'évaluation des programmes de formation professionnelle et technique sont encore trop rares en Afrique. Cette rareté mène à un manque de données sur le chômage des jeunes, une évaluation insuffisante des projets, des analyses pauvres ou inexistantes des programmes passés, et l'absence d'études d'impact. La création d'observatoires des formations professionnelles et du marché du travail peut aider à combler ces carences dans un certain nombre de pays africains. Ces observatoires peuvent soutenir I'utilisation efficace des fonds de formation et peuvent aider à identifier les bénéficiaires dont les besoins personnels et professionnels peuvent correspondre aux besoins du marché du travail. Leur méthode de travail est relativement simple : ils rassemblent des statistiques sur les évolutions du marché du travail et conduisent des enquêtes auprès de la population active comme des employeurs potentiels. Ils cadrent donc bien avec les moyens de la plupart des pays africains, et leurs contributions à la gestion prévisionnelle nationale, régionale et locale pourraient être considérables.

Leur valeur a été démontrée par la Banque africaine de développement et le Centre de développement de I'OCDE dans leur rapport commun, Perspectives économiques en Afrique. Le PEA montre que cinq ans après leur diplôme, environ un quart des anciens étudiants des centres de formations professionnelle et technique en Namibie se déclare sans emploi. En Zambie, au contraire, une étude similaire réalisée en 2004 montre que la majorité des étudiants de même type a trouvé un emploi après six mois.

Il est essentiel d'étudier les causes profondes du chômage des jeunes pour que les formations soient pertinentes avant que tout investissement massif ne soit entrepris. Cette observation, pourtant simple, est trop souvent ignorée.

Deux autres études restituées dans le rapport susmentionné montrent qu'à peine la moitié des 25 entreprises rwandaises interrogées était satisfaite des performances des anciens étudiants de ces formations. La haute autorité des formations du Botswana (Botswana Training Authority - BOTA) s'est rendue compte dans une étude de 2005 que les instituts de formation du pays ne produisaient pas des individus prêts pour l'emploi car les programmes ne prévoyaient pas de stage pratique. Des doutes similaires s'expriment en Namibie. Certains indicateurs montrent que de telles incohérences existent dans la plupart des pays africains.

Ces éléments renforcent l'argument en faveur d'études avec suivi des diplômés, d'enquêtes régulières sur les caractéristiques du marché du travail, et de prévisions des besoins en compétences spécifiques. Cette approche active du marché du travail permettrait aux gouvernements d'être mieux positionnés pour prévoir et réagir aux évolutions de la main-d'œuvre, concevoir des curriculums de formation et mettre en place des infrastructures adéquates via les secteurs privé et public.

* Il s'agit d'un rapport conjoint de la Banque africaine de développementet du Centre de développement de I'OCDE, avec la Commission Économique pour l'Afrique des Nations unies, qui a bénéficié du soutien financier de la Commission européenne.

Les lecteurs sont invités à citer ou reproduire les informations des Repères du Centre de développement de I'OCDE dans leurs propres publications. En échange, le Centre demande les remerciements de rigueur ainsi qu'un exemplaire de la publication. Le texte intégral des Repères et d'autres informations sur le Centre de développement et ses travaux sont disponibles sur : www.oecd.org/dev
Centre de développement de I'OCDE 2, rue André-Pascal, 75775 Paris Cedex 16, France Tél : $33(0) 145.24 .82 .00$

Fax : $33(0) 144.30 .61 .49$ mél : cendev.contact@oecd.org 